\title{
Cardiac arrest and catecholamine cardiomyopathy secondary to a misdiagnosed ectopic pheochromocytoma
}

\author{
Xiao Ying Chen, Xian Liu, Shijing Tian, Xi Wang, Hong Tang, Yingxi Yu, Dan Zhang, Yan Peng \\ Department of surgical intensive care unit, the First Affiliated Hospital of Chongqing Medical University, Chongqing, China
}

Key words: pheochromocytoma; cardiac arrest; catecholamine cardiomyopathy

A 47-year-old female was admitted for "abdominal distension with nausea and vomiting for eight days" and had no previous medical history, including hypertension or syncope. Abdominal computed tomography (CT) showed a $3.1 \mathrm{~cm} \times 3.7 \mathrm{~cm} \times 4.5 \mathrm{~cm}$ round mass shadow at the border between the descending and horizontal segments of the duodenum and behind the head of the pancreas on January 30, 2019 (Fig. 1). The round mass was misdiagnosed as "stromal tumour" and underwent laparoscopic resection on February 19, 2019. Blood pressure and heart rate increased sharply until ventricular fibrillation occurred when the tumour was touched with the instrument after general anaesthesia (Propofol/Remifentanil/Sevoflurane). The operation had to be stopped and cardiopulmonary resuscitation was performed. After cardiac recovery, left ventricular ejection fraction (LVEF) was $25 \%$ and fraction shortening (FS) was $12 \%$ by transthoracic echocardiography, and plasma catecholamines metabolites were significantly increased (m-norepinephrine $427.6 \mathrm{ng} / \mathrm{L}$, norepinephrine $360.2 \mathrm{ng} / \mathrm{L}$, vanilla-bitter almond acid (VMA) $213.2 \mathrm{ng} / \mathrm{mL}$, catecholamine $180.1 \mathrm{ng} / \mathrm{mL}$ ). The patient was diagnosed with catecholamine cardiomyopathy and cardiogenic shock, and then sent to surgical ICU (SICU) for PICCO monitoring and anti-shock treatment.

After the patient was sent to SICU, blood pressure was maintained with cardiac drugs (dopamine/dobutamine) and norepinephrine, but ECMO treatment was rejected by the patient's families. Variables (PICCO parameters/EF values/cTnT) of the first six days after surgery are shown in Table 1 . During cardiogenic shock our goal is to ensure that the level of lactic acid is $<2 \mathrm{mmol} / \mathrm{L}$, while mean arterial pressure and cardiac preload (GEDI) are as low as possible. Sustained ventricular tachycardia occurred on the first day and returned to sinus rhythm on the third day. Lidocaine

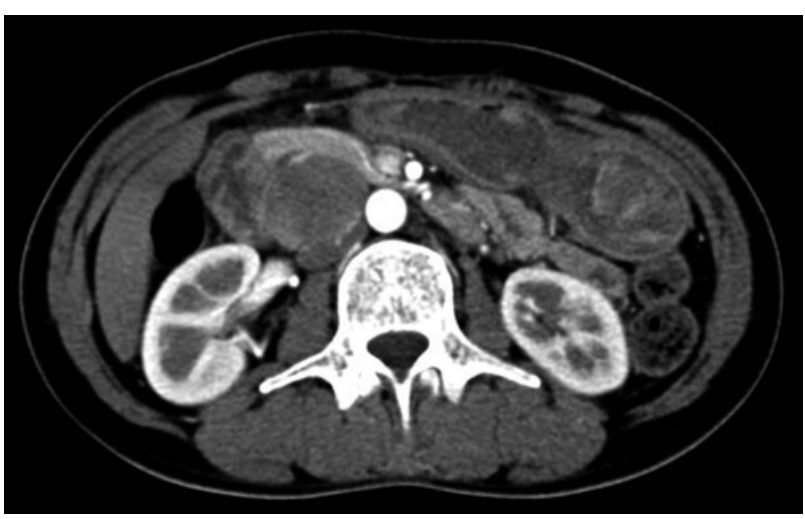

Figure 1. Abdominal computed tomography image

was not effective in correcting arrhythmia. On the $4-5^{\text {th }}$ day, the patient's LVEF began to improve significantly and mechanical ventilation was stopped. On the sixth day after surgery, LVEF and plasma catecholamine metabolite levels returned to normal, and cardiotonic agents were discontinued. The patient was discharged without any complications but still had symptoms of abdominal distension and progressive aggravation.

She was revisited on July 22, 2019. LVEF was $64 \%$ and plasma catecholamine metabolites were increased (m-norepinephrine $1119.6 \mathrm{ng} / \mathrm{L}$, norepinephrine 1949.5 $\mathrm{ng} / \mathrm{L}$, VMA $586.5 \mathrm{ng} / \mathrm{mL}$, catecholamine $369.3 \mathrm{ng} / \mathrm{mL}$ ). Abdominal CT showed that the mass was slightly larger than before, with a size of about $4 \mathrm{~cm} \times 3.5 \mathrm{~cm} \times 4.8 \mathrm{~cm}$. Iodine 131-labeled metaiodobenzylguanidine scintigraphy showed abnormal radioactivity accumulation in the right adrenal region at $24 \mathrm{~h}, 48 \mathrm{~h}$, and $72 \mathrm{~h}$. The patient underwent successful tumour resection on September 3, 2019 after two weeks of treatment with phenbenzamine (20 mg tid) and was confirmed as pheochromocytoma by pathological examination. 
Table 1. Variables of the first 6 days afte $r$ surgery

\begin{tabular}{|c|c|c|c|c|c|c|}
\hline Variables & Day 1 & Day 2 & Day 3 & Day 4 & Day 5 & Day 6 \\
\hline $\mathrm{Cl}\left[\mathrm{L} / \mathrm{min} / \mathrm{m}^{2}\right]$ & 1.98 & 2.05 & 2.09 & 2.82 & 3.05 & - \\
\hline GEDI $\left[\mathrm{mL} / \mathrm{m}^{2}\right]$ & 706 & 604 & 673 & 710 & 642 & - \\
\hline SVRI [dyn $\left.\quad \mathrm{s} / \mathrm{cm}^{-5} \quad \mathrm{~m}^{2}\right]$ & 3210 & 2447 & 2102 & 1921 & 1756 & - \\
\hline ELWI $[\mathrm{mL} / \mathrm{kg}]$ & 10.1 & 9.9 & 8.9 & 9.7 & 8.0 & - \\
\hline $\mathrm{EF}(\%)$ & 23 & 30 & 30 & 34 & 45 & 50 \\
\hline $\mathrm{cTnT}[\mu \mathrm{g} / \mathrm{L}]$ & 4.99 & 3.14 & 2.54 & 3.04 & 1.55 & 0.865 \\
\hline NT-proBNP [ng/L] & 7823 & 18300 & 26456 & 19359 & 12423 & 609 \\
\hline
\end{tabular}

$\mathrm{Cl}$ — cardiac index; GEDI — global end-diastolic volume index; SVRI — systemic vascular resistance index; ELWI — extra vascular lung water index; EF — ejection fraction; cTnT — cardiac troponin; NT-proBNB — N-terminal pro b-type natriuretic peptide

Pheochromocytoma is a rare neuroendocrine tumour, which can be acute and even life-threatening, and which is associated with the sudden release of large amounts of catecholamines [1]. The most common symptoms of pheochromocytoma are palpitations, hyperhidrosis, and headache [2], and a small number of patients will present with acute catecholamine cardiomyopathy [3], and life-threatening acute myocardial injury and dysfunction. Cardiogenic shock, circulatory failure, and multisystem damage are the main manifestations of catecholamine cardiomyopathy and are very likely to cause patient death. Therefore, early identification of pheochromocytoma, enhanced preoperative management, as well as prevention of acute catecholamine cardiomyopathy occurrence are essential [4].

Our patient was misdiagnosed as intestinal stromal tumour before the first operation and thus did not receive effective preoperative preparation, resulting in intraoperative acute catecholamine cardiomyopathy and sudden cardiac arrest. Myocardial toxicity from high circulating catecholamine production by pheochromocytoma causes cardiomyopathy in a minority of patients, manifesting as cardiac hypertrophy or dilated cardiomyopathy, which can be present at any age, including childhood. Echocardiography can visualise a decreased ejection fraction (EF) and wall motion abnormalities [5]. Our case did not present with cardiomyopathy, but hyperactive heart disease during surgery became the main manifestation, with an initial score of only $15 \%$, and recurrent ventricular fibrillation, a hyperactive catecholamine cardiomyopathy that is rarely reported.

As can be observed in our case, acute catecholamine cardiomyopathy is reversible, and the left ventricular fraction recovers to $45 \%$ after $3 \sim 4$ days. The focus of treatment is tantamount to correct tissue hypoperfusion at the stage of cardiogenic shock, while avoiding exces- sive fluid and increasing the burden on the heart. Accurate haemodynamic monitoring (such as PICCO/cardiac ultrasound) and ECMO can help the rehabilitation of patients. Although there are also examples of successful surgery without preoperative drug preparation, we still recommend preoperative medication due to the potential possibility of acute catecholamine cardiomyopathy and sudden cardiac arrest.

\section{Authors' contribution}

X.Y.C. and X.L. contributed equally to this work and should be considered as co-first authors. X.C. and X.L. proposed and wrote the study. All authors contributed to the intellectual content and approved the final version.

\section{Funding}

None.

\section{Conflicts of interest}

The authors declare that there are no financial interests or potential conflicts of interest relating to the manuscript.

\section{References}

1. Lenders J, Eisenhofer G, Mannelli M, et al. Phaeochromocytoma. Lancet. 2005; 366(9486): 665-675, doi: 10.1016/s0140-6736(05)67139-5, indexed in Pubmed: 16112304 .

2. Prejbisz A, Lenders J, Eisenhofer G, et al. Cardiovascular manifestations of phaeochromocytoma. J Hypertens. 2011; 29(11): 2049-2060, doi: 10.1097/hjh.0b013e32834a4ce9, indexed in Pubmed: 21826022.

3. Giavarini A, Chedid A, Bobrie G, et al. Acute catecholamine cardiomyopathy in patients with phaeochromocytoma or functional paraganglioma. Heart. 2013; 99(19): 1438-1444, doi: 10.1136/heartjnl-2013-304073, indexed in Pubmed: 23837998.

4. Pisarska M, Pędziwiatr M, Budzyński A, et al. Perioperative hemodynamic instability in patients undergoing laparoscopic adrenalectomy for pheochromocytoma. Gland Surg. 2016; 5(5): 506-511, doi: 10.21037/gs.2016.09.05, indexed in Pubmed: 27867865.

5. Jia X, Guo X, Zheng Q, et al. Perioperative management of paraganglioma and catecholamine-induced cardiomyopathy in child - a case report and review of the literature. BMC Anesthesiology. 2017; 17(1): 142, doi: 10.1186/s12871-017-0433-0, indexed in Pubmed: 29041921. 